\title{
Ameliorating Effect of Oral Guggulsterone Administration in Imiquimod-Induced Psoriasis in Mice Raghad A. Khaleel ${ }^{*}$ and Munaf H. Zalzala**,1
}

* Department of Pharmacology, College of Medicine, University of Iraqia, Baghdad. Iraq.

** Department of Pharmacology and Toxicology, College of pharmacy, University of Baghdad, Baghdad. Iraq.

\begin{abstract}
Psoriasis is a common chronic skin condition characterized by infiltration of inflammatory cells into the epidermis and altered keratinocyte differentiation.

In this work, psoriasis was induced by an imiquimod 5\% cream, an immune response modifier that can induce psoriasis-like skin inflammation when applied topically in mice. Guggulsterone prepared as a suspension and has been orally given to mice before imiquimod application. The results of the current study showed that guggulsterone suspension can significantly reduce psoriasis area and severity index in (guggul suspension + imiquimod group as compared with both control group and (vehicle suspension + imiquimod ) group.
\end{abstract}

Keywords: Psoriasis, Guggulsterone, Imiquimod5\%, NF-kB, TNF- $\alpha$, IL-23, IL17.

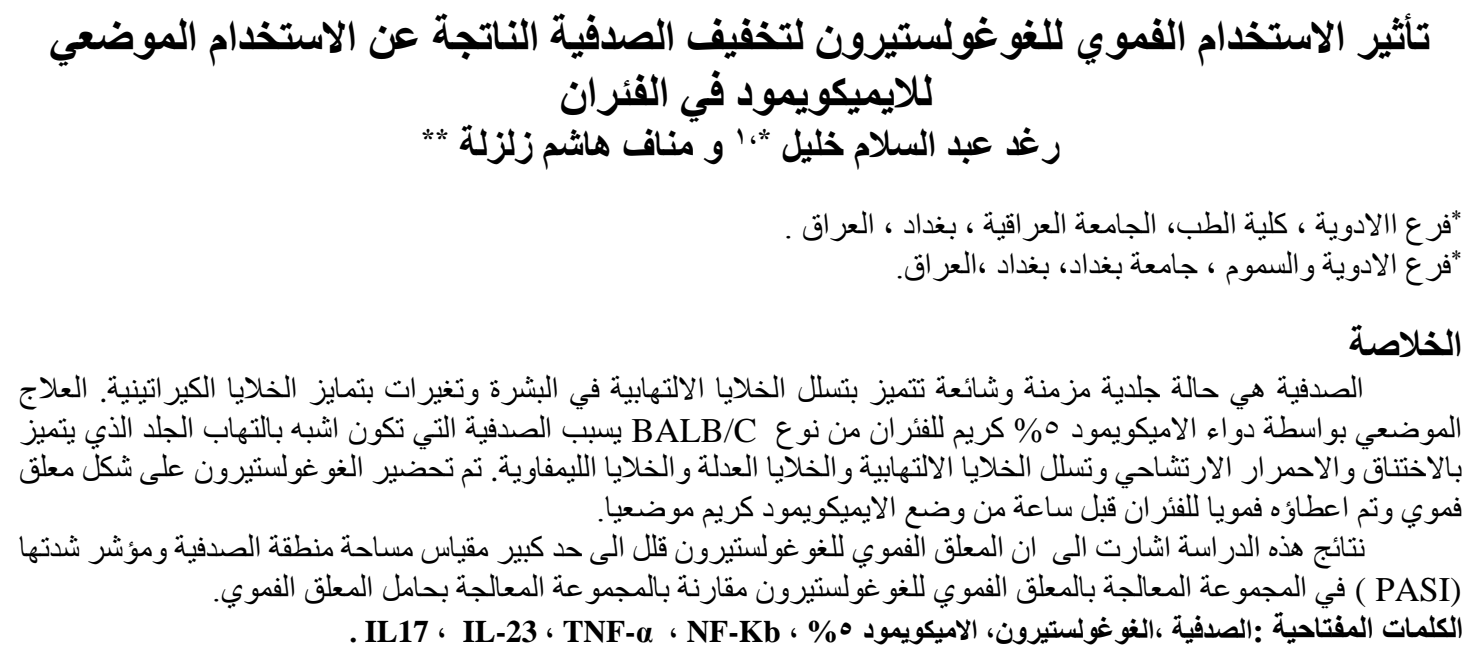

\section{Introduction}

Psoriasis is a common chronic skin condition characterized by infiltration of inflammatory cells into the epidermis and altered keratinocyte differentiation. It is currently thought of as at-cell mediated type-1 autoimmune disease ${ }^{(1)}$.

Psoriasis is believed to result from a complex interplay between genetics, environment, and skin barrier disruption, and immune dysfunction $^{(2,3)}$.

Psoriasis exists on a reasonably broad spectrum in terms of clinical manifestations and it is associated with a higher percent of morbidity and the current medications can have acute side effects. Numerous clinical studies have reported a strong relation between psoriasis and atherosclerosis, type 2 diabetes, and metabolic syndrome, together with the leading causes of mortality in the Western world ${ }^{(4)}$.

${ }^{1}$ Corresponding author E-mail: munafzalzala@copharm.uobaghdd.edu.iq Received: $1 / 8 / 2018$ Accepted: 4/9/2018

Iraqi Journal of Pharmaceutical Sciences 
Also, patients with psoriasis-like those with other major medical disorders, have decreased levels of employment and income as well as reduced quality of life ${ }^{(5)}$. The combined costs of long-term therapy and social costs of the disease have a significant impact on health care systems and on the society in general ${ }^{(6)}$.

The intended skin disease is characterized by thickened, scaly skin patches or psoriatic plaques, caused by abnormal keratinocyte proliferation and infiltration of inflammatory cells into the dermis and epidermis. About $10-30 \%$ of patients with psoriasis also develop psoriasis arthritis. Once psoriasis appears, it is usually a life-long disease $^{(2)}$.

Psoriasis can be triggered by a number of factors that includes physical injury to the skin (the 'Koebner' response), administration of interferon (IFN) or other inflammationinducing stimuli, rapid withdrawal of immunesuppressive drugs like corticosteroids and (systemic) infections with streptococcus or other bacteria ${ }^{(2)}$.

Hyper proliferation and abnormal differentiation of keratinocytes are the two important results of the underlying pathophysiologic dysregulation in psoriasis. Histologically, there is distinctive thickening of the epidermis due to increased proliferation of keratinocytes in the inter follicular epidermis, and an epidermal rete - downward undulations of the epidermis - become very elongated ${ }^{(7,8)}$.Keratinocyte differentiation is largely altered in psoriasis, paralleling 'regenerative maturation,' an alternative cell differentiation program that is transiently expressed during wound repair. The granular part of the epidermis in which terminal differentiation starts is highly decreased or absent in psoriatic lesions ${ }^{(8,9)}$.Consequently a stratum corneum forms from incompletely differentiated keratinocytes that irregularly retain a cell nucleus (parakeratosis). Histological features of plaque psoriasis are the loss of the granular layer, supra papillary thinning, microabscess of Munro (collection of neutrophils in the stratum corneum ), spongi form pustule of Kojog (epidermal spongiotic pustule with neutrophilic infiltration), dermal/epidermal CD3 $+\mathrm{T}$ cell infiltrates with the epidermotropisim of CD8+ T cells . The histology of psoriatic subtypes may share classic dermal and epidermal features of a plaque psoriasis however, they may also display marked histopathologic features $(10,11)$.

There are at least three distinct inflammatory pathways that may drive distinct clinical phenotypes in psoriasis: chronic plaque-type psoriasis by tumour necrosis factor
(TNF), acute psoriasis by type I IFNs, and pustular psoriasis via IL - 36 / IL-1.

However, it is likely that the separation of these phenotypes and pathways is not absolute but instead represent the far end of a spectrum. Hence, specific phenotypes may contain variable amounts of cytokines of this different inflammatory path- ways at a given time ${ }^{(12)}$.

Imiquimod (IMQ) (Aldara cream 5\%) is an immune response modifier was first approved by US Food and Drug Administration (FDA) ${ }^{(13)}$ for the treatment of external genital and perianal warts in 1997 and then consecutively approved for actinic keratosis (AK) and superficial basal cell carcinoma $(\mathrm{BCC})^{(14)}$.

Topical treatment of IMQ in BALB/c mice induced psoriasis-like skin dermatitis distinguished by acanthosis, erythema, and inflammatory infiltrate combined with neutrophils, dendritic cells (DCs) and lymphocytes ${ }^{(15)}$. Daily topical application of IMQ on the skin of mice can lead to psoriasislike dermatitis with many hallmarks of human psoriases, such as the formation of microabscesses, skin thickening, hyperkeratosis, acanthosis, scaling, and erythema ${ }^{(16)}$.

Guggulsterone (GS) [4,17(20)pregnadiene-3,16-dione] is the plant-derived steroid desolated from the gum resin of the Commiphora Mukul tree, named guggulipid ${ }^{(13)}$. It is a polyphenol conventionally used to treat obesity, diabetes, hyperlipidemia, atherosclerosis, and osteoarthritis, possibly through an anti-inflammatory mechanism ${ }^{(17)}$. The active substances in Guggulipid are the pregnane plant sterols cis-guggulsterone (EGS) and trans-guggulsterone (Z-GS). Guggulsterone (GS) has anticancer potential as indicated by its ability to suppress the proliferation of a broad types of human tumor cell lines ${ }^{(18)}$.

The anti-arthritic and anti-inflammatory activity of gum guggul was established as early as 1960 , by Gujral et al ${ }^{(19)}$. The effectiveness of guggul for treating osteoarthritis of the knee has also been demonstrated; furthermore, it has shown that guggulsterone is an antagonist for bile acid receptor farnesoid $\mathrm{X}$ receptor ${ }^{(20)}$. Other study has been shown that guggulsterone can augment transcription of the bile salt export pump, thus regulating cholesterol homeostasis; moreover, nuclear factor kappa-light-chainenhancer of activated B cells $(\mathrm{NF}-\kappa \mathrm{B})$ has been implicated in obesity, hyperlipidemia, atherosclerosis, osteoarthritis, and bone loss, all of which can be modulated by guggulsterone ${ }^{(21)}$. 
In the present study, guggulsterone was prepared as a suspension and given orally to mice before psoriasis induction via imiquimod.

\section{Materials and Methods}

\section{Chemicals and kits}

The chemicals that were used in this work includes formaline(merk chemicals ,Germany ) , diethylether (BDH chemicals, india ), xanthane as asuspending agent(Samara drug industry Iraq), Guggulsterone (Xi'an geekee biotech, China).

The kits used in this study include (Interleukin-23, Interleukin-17, Tumor necrosis factor alfa,nuclear factor kappa B)all of them from (Elab .science, China)

Methods

Animal treatment

Twenty one (Albino, BALB/c mice male, 8 weeks) (weighing between 25 to $40 \mathrm{gm}$ ) purchased from the department of drug control.

They were kept in the animal house of college of pharmacy/University of Baghdad under specific pathogen-free conditions and provided with water and food ad libitum under 12-hr light-dark cycle and maintained conventionally during the study with regulated air temperature $\left(15-21^{\circ} \mathrm{C}\right)$.

\section{Preparation of guggulsterone suspension}

Guggulsterone suspension is prepared by making a thick paste in mortar composed of $0.5 \mathrm{gm}$ guggulsterone powder and $0.09 \mathrm{gm}$ methylparaben and $0.015 \mathrm{gm}$ propyl paraben and $5 \mathrm{ml}$ glycerine as dispersing agent, and use xanthan $0.25 \mathrm{gm}$ as suspending agent and then complete the volume to $5 \mathrm{ml}$ using distilled water (final concentration of suspension is $0.5 \%)^{(22)}$.

\section{Experimental protocoal}

In this work we have weighed approximately twenty-one mice at zero time and we measured ear thickness using vernier at zero time and we shave the back area of all mice using traditional manual eraser (trimming not zero shaving).

Mice were divided into three groups (seven mice each) as follow:

1- Control group in which mice were received vehicle of gugglsterone suspension daily for 14 consecutive days.

\section{2-Imiquimod group (vehicle} suspension+IMQ) in which mice were received daily oral gavage of $0.2 \mathrm{ml}$ of vehicle for Gugglsterone suspension one hour before imiquimod 5\% application for 14 consecutive days. 3-Guggulsterone-treated
(guggulsterone suspension+IMQ) in which mice were received daily oral gavage of $0.2 \mathrm{ml}$
Gugglsterone suspension one hour before imiquimod 5\% application for 14 consecutive days.

The experiment last for 14 consecutive days, at the end of our experiment (day 14), psoriasis area and severity index was determined carefully by a professional dermatologist, and all mice were weighed before killing, and finally mice were sacrificed, and blood collected for biochemical analysis, also skin of back area and right ear were taken for histopathological examination, the spleen was weighed and spleen index was calculated.

\section{Scoring severity of skin inflammation}

To score the severity of inflammation of the back skin, the Psoriasis Area and Severity Index (PASI) score for back skin was determined by a professional dermatologist. Erythema, scaling, and thickening was scored independently on a scale from 0 to 4 : 0 , none; 1, slight; 2, moderate; 3 , marked; 4, very marked. The cumulative score (erythema plus scaling plus thickening) served as a measure of the severity of inflammation (scale 0-12) ${ }^{(23)}$.

\section{Measurements of spleen index}

At the end of the experiment (day 14), the body weight and spleen weight for all mice was measured and the spleen index was calculated by dividing spleen weight in $\mathrm{mg}$ by the body weight that was measured during sacrificing time in gm $^{(24)}$.

\section{Measurements of ear thickness}

The right ear thickness in mice was measured using digital vernier caliper every other day ${ }^{(25)}$.

\section{Measurements of skin thickness}

After sacrificing our mice, back skin thickness was measured in $\mathrm{mm}$ via digital vernier caliper ${ }^{(26)}$ and comparing the measured thickness among groups.

\section{Preparation of serum sample}

After euthanization of the animal by anesthetic diethyl ether, blood was collected by heart puncture and put in an Eppendorf tube and centrifuged at 3500rpm for 15 minutes to obtain serum, which was separated and stored at $-20 \mathrm{C}$ until the day of analysis. Serum was utilized for estimation of IL-23 and IL-17 ${ }^{(27)}$.

\section{Preparation of skin tissue homogenate}

At the end of the experiment (day 14), the skin from the back area was taken by incision and washed by phosphate buffer $\mathrm{pH}$ (77.2). Ten percent (10\%) of back skin tissue homogenate was prepared and centrifuged at $5000 \mathrm{rpm}$ for 15 minutes, and the supernatant is stored at $-20{ }^{\circ} \mathrm{C}$ until the day of analysis. Skin homogenate was utilized to determine the TNF$\alpha$ and NF-Kb level in tissue ${ }^{(28)}$. 


\section{Histological examination}

Skin from back area and right ear utilized in this study were prepared for histological examination according to the method of Junqueira et al at 1995 using paraffin section methods ${ }^{(29)}$.

\section{Statistical analysis}

The significance of differences between the mean values was calculated using paired and unpaired student t-test. P-value less than 0.05 were considered significant for all data shown in this respect ${ }^{(30)}$.

\section{Results}

Effect of oral administration of guggulsterone on the PASI score in imiquimod-induced psoriasis in mice.

Table 4: showed that there was a significant decreament in the measured psoriasis area and severity index in PASI score in (vehicle susp+IMQ) group as compared to the control group. In (guggul susp+IMQ) group, the oral administration of guggulsterone produces a reduction in PASI score significantly in comparison with (vehicle susp+IMQ) group.

Table 1. The psoriasis area and severity index (PASI) score after oral guggulsterone administration in imiquimod-induced psoriasis in mice.

\begin{tabular}{|c|c|c||}
\hline $\begin{array}{c}\text { Control } \\
\text { group }\end{array}$ & $\begin{array}{c}\text { ( vehicle } \\
\text { susp+IMQ) } \\
\text { group }\end{array}$ & $\begin{array}{c}\text { (Guggul } \\
\text { susp+IMQ) } \\
\text { group }\end{array}$ \\
\hline Zero & $5.55 \pm 2.23^{*}$ & $1.2 \pm 1.35^{\#}$ \\
\hline
\end{tabular}

- Each value represents mean \pm standard deviation (SD).

- *= Significantly different $(P<0.05)$ with respect

to the negative control group.

- \# $\mathrm{P}<0.05 \%$ significant in comparison with vehicle susp + IMQ group.

- $\mathrm{N}=$ number of animals.

\section{Effect of oral administration of} guggulsterone on the ear thickness in imiquimod-induced psoriasis in mice.

Figure 1 showed that there was very slight increase in ear thickness in (guggul susp + IMQ) group as compared with control group, in (vehicle susp+IMQ) group there was a significant increment in ear thickness as compared with control group.

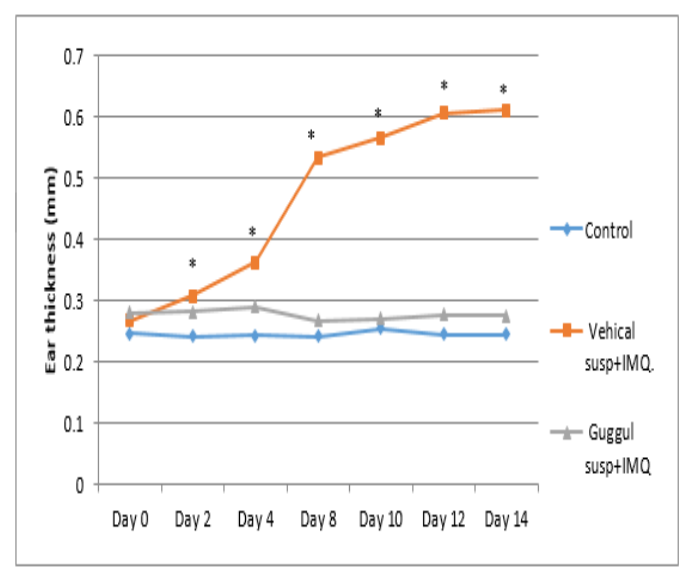

Figure 1. Effect of oral administration of guggulsterone on ear thickness in imiquimod-induced psoriasis in mice.

- Each value represents mean \pm standard deviation (SD).

- *= Significantly different $(P<0.05)$ with respect to the negative control group.

- \# $\quad \mathrm{P}<0.05 \%$ significant in comparison with vehicle susp + IMQ group.

\section{Effect of oral administration of guggulsterone on the skin thickness in imiquimod-induced psoriasis in mice.}

Figure 2 showed that the oral administration of guggulsterone produce significant reduction in skin thickness in compare with (vehicle susp + IMQ) group. Oral administration of guggulsterone successful in produce about $20 \%$ reduction in skin thickness after imiquimod administration.

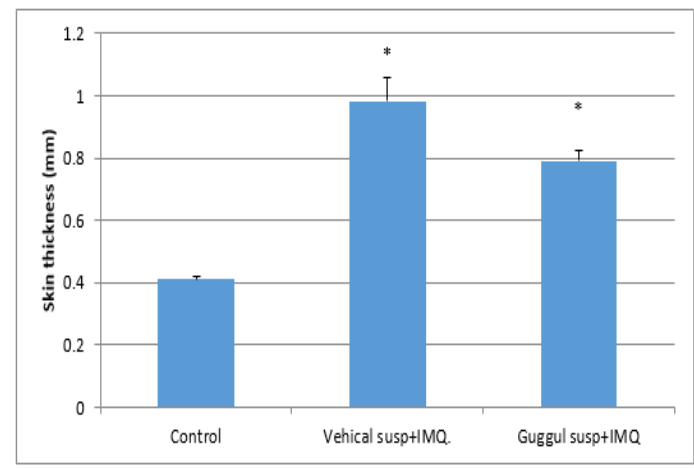

Figure 2. Effect of oral administration of guggulsterone on skin thickness in imiquimod-induced psoriasis in mice.

- Each value represents mean \pm standard deviation (SD).

- *= Significantly different $(P<0.05)$ with respect to the negative control group.

- \# $\quad \mathrm{P}<0.05 \%$ significant in comparison with vehicle susp+IMQ group 
Effect of oral administration of guggulsterone on the spleen index in imiquimod-induced psoriasis in mice.

Figure 3 showed that there was obvious increment in the measured spleen index in (vehicle susp+IMQ) group as compared with control group, while in (guggul susp+IMQ)group there was marked elevation in the measured spleen index as compared with control group.

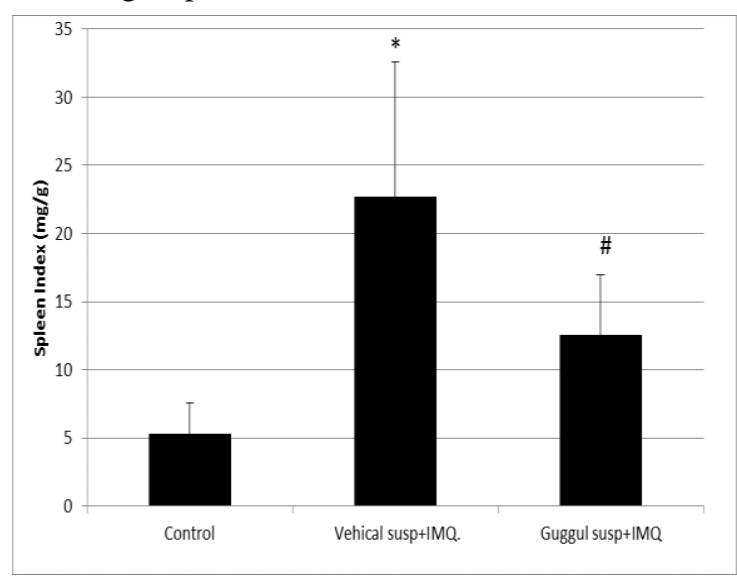

Figure 3. Spleen index after oral administration of guggulsterone in imiquimod - induced psoriasis in mice.

- Each value represents mean \pm standard deviation (SD).

- *= Significantly different $(P<0.05)$ with respect to the negative control group.

- \# $\quad \mathrm{P}<0.05 \%$ significant in comparison with vehicle susp+IMQ group.

Effect of oral administration of guggulsterone on serum IL-23 level in imiquimod-induced psoriasis in mice.

Figure 4,5,6,7: showed that there was no significant change in serum IL-23 level in (vehicle susp+IMQ) group $(\mathrm{P}<0.05)$ as compared with normal control group, also there is no significant elevation in serum IL-23 level $(\mathrm{P}>0.05)$ in (guggul susp+IMQ) group as compared to normal control group, regarding serum IL-17 level, there was a significant elevation in serum IL-17 level in (vehicle susp+IMQ) group $(\mathrm{P}<0.05)$ in comparison with normal control group, also there was significant elevation in serum IL-17 level $(\mathrm{P}<0.05)$ in (guggul susp+IMQ) group as compared to normal control group.

Relatively, there was a significant elevation in tissue NF-KB level in (vehicle susp+IMQ) group $(\mathrm{P}<0.05)$ as compared with normal control group,also there was significant elevation in serum NF-KB level in $(\mathrm{P}<0.05)$ (guggul susp+IMQ) group as compared to normal control group. Finally there was significant elevation in tissue TNF- $\alpha$ level in (vehicle susp+IMQ) group $(\mathrm{P}<0.05)$ compared with control group, also there was significant elevation in tissue TNF- $\alpha$ level $(\mathrm{P}<0.05)$ in (guggul susp+IMQ) group as compared to normal control group.

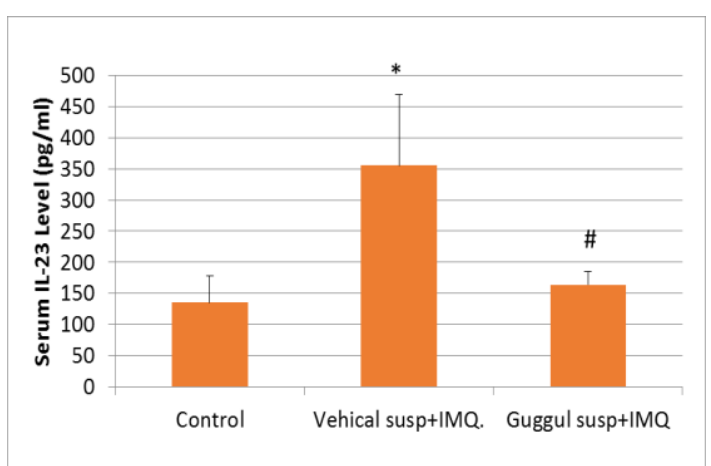

Figure 4. Effect of oral administration of guggulsterone on serum IL-23 level in imiquimod-induced psoriasis in mice.

- Each value represents mean \pm standard deviation (SD).

- *= Significantly different $(P<0.05)$ with respect to the negative control group.

- \# $\quad \mathrm{P}<0.05 \%$ significant in comparison with vehicle susp+IMQ group.

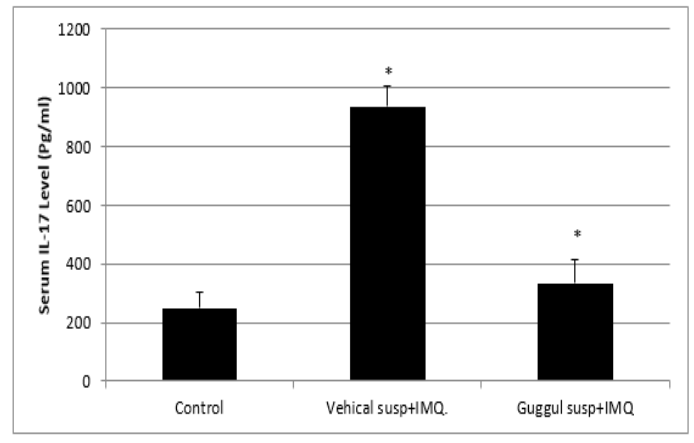

Figure 5. Effect of oral administration of guggulsterone on serum IL-17 level in imiquimod-induced psoriasis in mice.

- Each value represents mean \pm standard deviation (SD).

- *= Significantly different $(P<0.05)$ with respect to the negative control group.

- \# $\quad \mathrm{P}<0.05 \%$ significant in comparison with vehicle susp+IMQ group. 


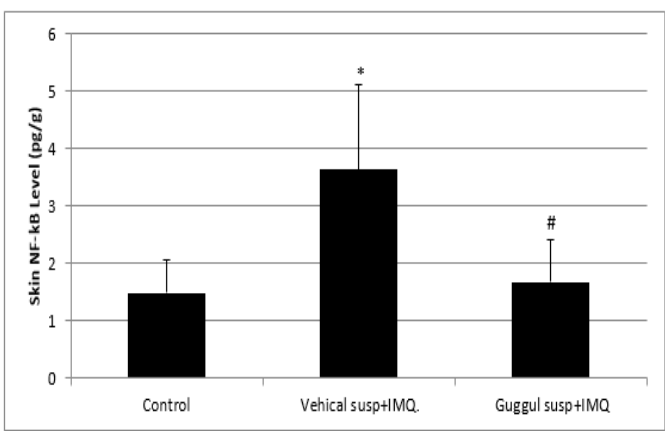

Figure 6. Effect of oral administration of guggulsterone on tissue NF-kB level in imiquimod-induced psoriasis in mice.

- Each value represents mean \pm standard deviation (SD).

- *= Significantly different $(P<0.05)$ with respect to the negative control group.

- \# $\quad \mathrm{P}<0.05 \%$ significant in comparison with vehicle susp+IMQ group.

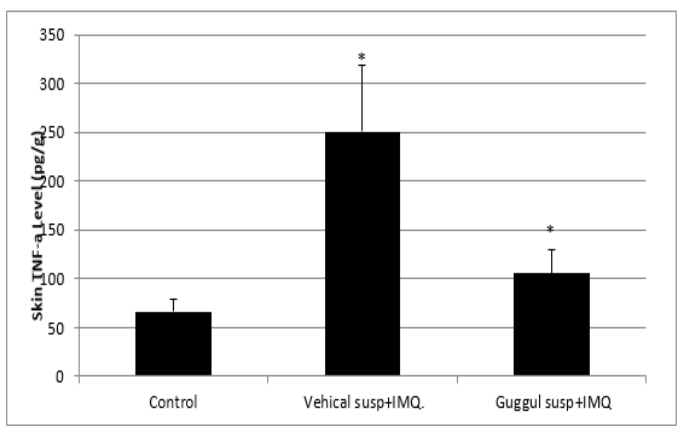

Figure 7. Effect of oral administration of guggulsterone on tissue TNF- $\alpha$ level in imiquimod - induced psoriasis in mice.

- Each value represents mean \pm standard deviation (SD).

- *= Significantly different $(P<0.05)$ with respect to the negative control group.

- \# $\quad \mathrm{P}<0.05 \%$ significant in comparison with vehicle susp+IMQ group.

\section{Histological examination of mice skin and right ear section.}

Sections of the back area and right ear look-like normal in appearance in control group in which the thickness of the epidermal layer look-like normal and no rete-ridge appear as in figure (1A and $\mathrm{B})$.

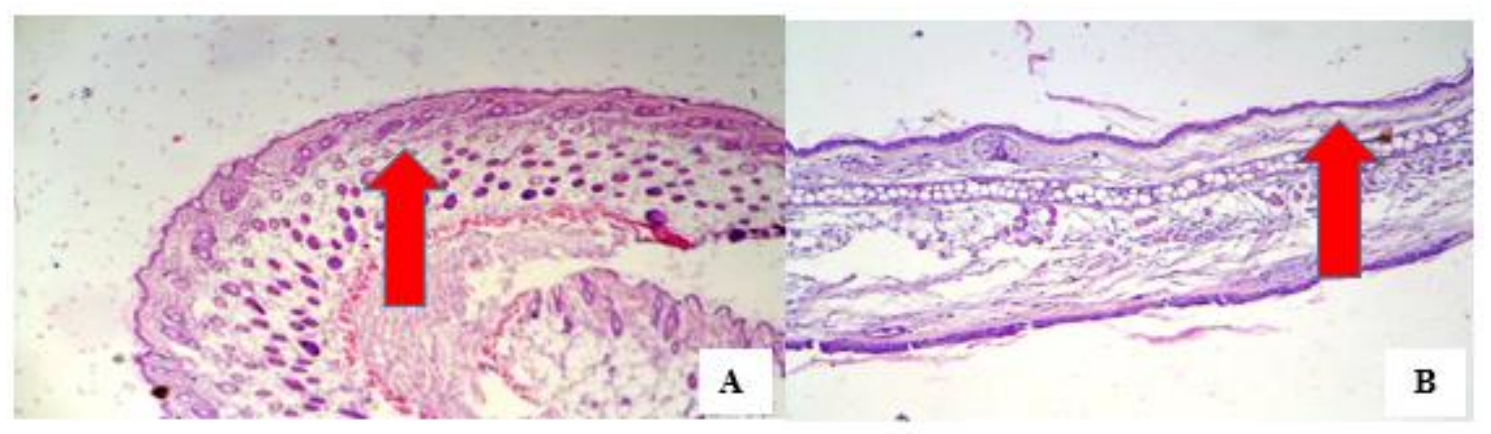

Figure 1. (H and E) staining sections of the mouse back area skin and ear skin of control group (200X) A: section of normal right ear B: section of the back area.

Red arrows : sign to the thickness of the epidermal layer of back area and right ear section.

In the (vehicle susp + IMQ) group section of back area skin and right ear showed slight acanthosis of stratified epithelium more than 6 cells with an increase of rete ridge as in Figure (2A and $\mathrm{B})$. 


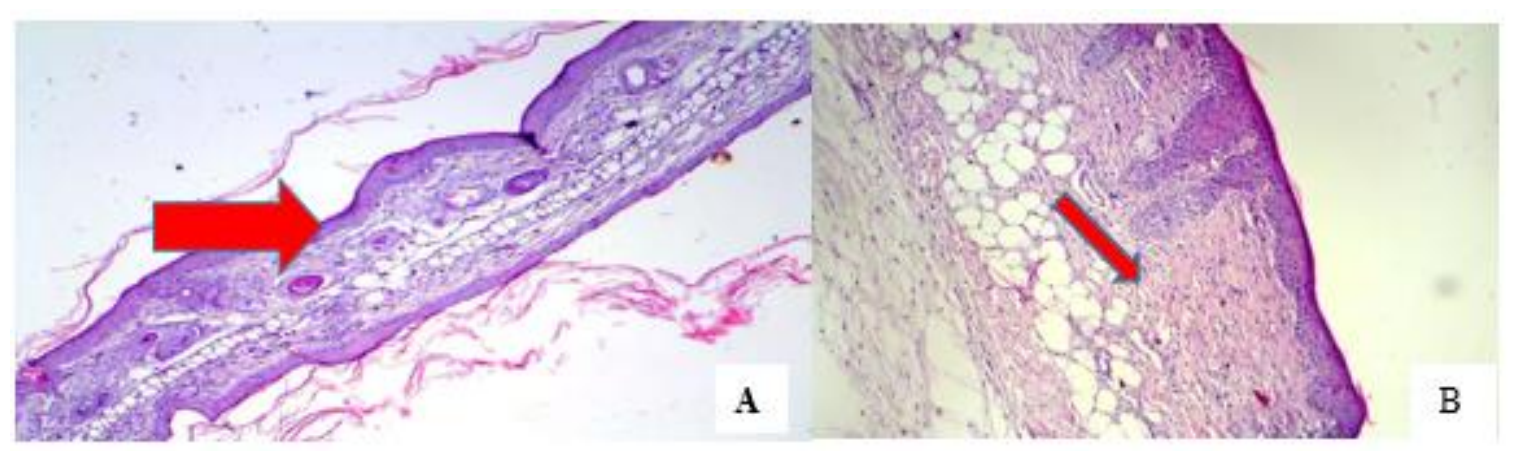

Figure 2. ( $\mathrm{H}$ and $\mathrm{E}$ ) staining sections of the mouse back area skin and ear skin of vehicle susp+IMQ group (200X)

A: section of back area skin B: section of right ear vehicle susp+IMQ group showed slight acanthosis of stratified epithelium more than 6 cells with an increase of rete ridge. Red arrows: sign to the thickness of the epidermal layer of back area and right ear section.

The histological examination of the back area and right ear sections in (guggul susp+IMQ) group look - like normal in appearance and no rete-ridge appear, and thickness of stratified epithelium (4 cells layer) as in (Figure 3A and B).
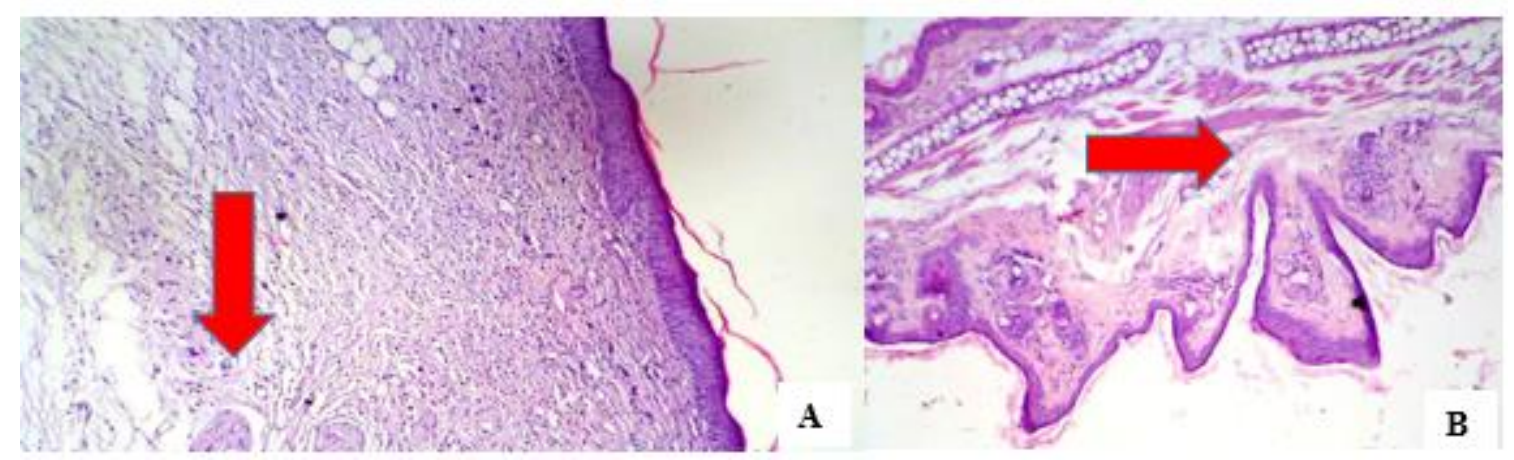

Figure 3. (H and E) staining sections of the mouse back area skin and ear skin of (IMQ+ guggul oint) group (200X).

A: Section of right ear B: Section of the back area. Red arrows: sign to the thickness of the epidermal layer of back area and right ear section.

\section{Discussion}

Psoriasis is a common skin disease that has been recognized since ancient times when it was erroneously thought to be a variant of leprosy. Psoriasis affects about 25 million people in North America and Europe, and is probably the most prevalent immune-mediated skin disease in adults. It is an organ-specific autoimmune skin disease that is triggered by an activated cellular immune system ${ }^{(7)}$ and is similar to other immune-mediated diseases such as Crohn's disease, rheumatoid arthritis, multiple sclerosis and juvenile-onset diabetes. All of these fit the definition of an autoimmune disease as a clinical syndrome caused by the activation of $\mathrm{T}$ cells and $\mathrm{B}$ cells, or both, in the absence of an ongoing infection or other discernable cause ${ }^{(7)}$.
In this work, psoriasis has been induced like skin inflammation in mice in both the back area and the right ear for 14 consecutive days by using imiquimod. Imiquimod ( IMQ) is a ligand for

TLR7 and TLR8 and a potent immune activator it is used for topical treatment of genital and perianal warts caused by human papillomavirus (31). IMQ application led to increased ear inflammation as evidenced by increased ear thickness ${ }^{(16)}$. Skin inflammation was also confirmed by histological examination which showed acanthosis, hyperkeratosis, and elongation of the rete-like ridge in the IMQ treated $\operatorname{group}^{(16)}$.

In 2016 Zhang et al found that IMQ could induce splenomegaly through systemic effects $^{(24)}$. 
Topical treatment with IMQ resulted in the rapid mobilization of $\mathrm{pDC}$ and high levels of type I IFN

in the blood and splenomegaly ${ }^{(32)}$, our results corresponds with this study in which we found that guggulsterone affects spenomegally induced by imiquimod.

More than that our findings regarding serum interleukin ( IL17,IL23) level showed that serum IL-17,IL-23 level were significantly reduced in (guggul susp+IMQ) group as compared with control group which indicate that guggulsterone suspension act as antiinflammatory agent by decreasing serum level of these interleukins.

It was postulated that IL-23 which is a cytokine driving the development of IL-17- and IL-22-producing Th17 cells, is functionally involved in the pathogenesis of psoriasis. Expression of IL-23 is increased in psoriasis lesional skin and increased numbers of Th17 cells are present. Guggulsterone suppressed NF-kB activated by carcinogens (phorbol ester, okadaic acid, and cigarette smoke condensate) and inflammatory stimuli (hydrogen peroxide, $\mathrm{TNF}$, and interleukin-1through inhibition of IKK, I B phosphorylation, and I kB degradation, which led to abrogation of p65 phosphorylation and nuclear translocation. Our findings regarding tissue $\mathrm{Nf}-\mathrm{Kb}$ showed that guggulsterone reduce $\mathrm{Nf}-\mathrm{kB}$ in orally treated (guggul susp+IMQ) groups as compared to (vehicle susp+IMQ).

Guggulsterone was quite effective in blocking the phosphorylation translocation of TNF-induced p65, which led to the abrogation of NFkB- dependent reporter gene expression. TNF-alfa level in orally treated groups showed a significant reduction in (guggul susp+IMQ) group as compared to (vehicle susp+IMQ) group in which (P-value $<0.05 \%$ ).

Various cell types in the psoriatic skin produce TNF and IL-17. TNF and IL-17 can stimulate neutrophil recruitment on their own, but also together in a synergistic manner, leading to enhanced inflammation. Psoriasis and other chronic inflammatory diseases seem to result from an overactive immune system with TNF, type I IFNs and the IL-23/IL-17 axis playing interwoven roles ${ }^{(33)}$. Histopathological examination of skin section and right ear indicated thatIMQ induces local psoriasis-like symptoms in susceptible humans and in mice, such as inflammation, thickening and scaling of the skin, epidermal hyperplasia with the parakeratosis (retention of nuclei in the stratum corneum), a neutrophil-rich stratum corneum (Munro's abscess), thinning of a granular layer of the epidermis, and a mononuclear dermal and epidermal infiltrate. Infiltration of $\mathrm{T}$ cells and dendritic cells in plaques and dysregulated angiogenesis of cutaneous blood vessels have also been described ${ }^{(34)}$.

\section{Conclusion}

All the results demonstrate that guggulsterone suspension had antiinflammatory effects. It is a promising intervention in psoriasis in future. Overall, our results indicate that guggulsterone apparently suppress NF-KB, TNF- $\alpha$ IL-17, IL-23 level, reducing (ear and skin thickness, spleen index) which may explain its anti-inflammatory activity.

\section{References}

1. Papp K, Berth-Jones J, Kragballe K, Wozel G, de la Brassinne M. Scalp psoriasis: A review of current topical treatment options. J Eur Acad Dermatology Venereol. 2007;21(9):1151-60.

2. Liu Y, Krueger JG, Bowcock AM. Psoriasis: Genetic associations and immune system changes. Genes Immun. 2007;8(1):1-12.

3. Chandran V, Raychaudhuri SP. Geoepidemiology and environmental factors of psoriasis and psoriatic arthritis. J Autoimmun. 2010;34(3):J314-21.

4. Gelfand JM, Neimann AL, Shin DB, Wang $\mathrm{X}$, Margolis DJ, Troxel AB. Risk of Myocardial Infarction in Patients With Psoriasis. Jama. 2006;296(14):1735.

5. Gelfand JM, Feldman SR, Stern RS, Thomas J, Rolstad T, Margolis DJ. Determinants of quality of life in patients with psoriasis: A study from the US population. J Am Acad Dermatol. 2004;51(5):704-8.

6. Guideline C. Assessment and management of psoriasis Clinical Guideline. Natl Clin Guidel Cent. 2012;(October):1-760.

7. Lowes MA, Bowcock AM, Krueger JG. Pathogenesis and therapy of psoriasis. Nature. 2007;445(7130):866-73.

8. Afifi $\mathrm{T}$, de Gannes G, Huang C, Zhou Y. Topical therapies for psoriasis: Evidencebased review. Can Fam Physician. 2005;51(APR.):519-25.

9. Laggner U, Meglio P Di, Perera GK, Lacy KE, Ali N, Smith CH, et al. Identification of a Novel Proinflammatory Human SkinHoming $\mathrm{V} \gamma 9 \mathrm{~V} \delta 2 \mathrm{~T}$ Cell Subset with a Potential Role in Psoriasis. 2015;

10. Ragaz et al.Histological features of psoriasis(March):1979(2)229_240.

11. Michelle A. Lowes, Mayte S. and J. G.K. IY32CH08-Lowes-Krueger Immunology of Psoriasis. Annu Rev. Immunol. 2014;32:227-55.

12. Conrad C, Gilliet M. Psoriasis: from Pathogenesis to Targeted Therapies. Clin 
Rev Allergy Immunol. 2018;54(1):102-13.

13. Mencarelli A, Renga B, Palladino G, Distrutti E, Fiorucci S. The plant sterol guggulsterone attenuates inflammation and immune dysfunction in murine models of inflammatory bowel disease. 2009;78:1214-23.

14. Miller RL, Gerster JF, Owens ML, Slade HB, Tomai MA. Review article Imiquimod applied topically: A novel immune response modifier and new class of drug. Int $\mathbf{J}$ Immunopharmacol. 1999;21(1):1-14.

15. OuYang Q, Pan YQ, Luo HQ, Xuan CX, Liu JE, Liu J. MAD ointment ameliorates Imiquimod-induced psoriasiform dermatitis by inhibiting the IL-23/IL-17 axis in mice. Int Immunopharmacol . 2016;39:369-76.

16. Nadeem A, Al-Harbi NO, Al-Harbi MM, El-Sherbeeny AM, Ahmad SF, Siddiqui N, et al. Imiquimod-induced psoriasis-like skin inflammation is suppressed by BET bromodomain inhibitor in mice through RORC/IL-17A pathway modulation. Pharmacol Res. 2015;99:248-57.

17. Shishodia S, Sethi G, Ahn KS, Aggarwal BB. Guggulsterone inhibits tumor cell proliferation, induces S-phase arrest, and promotes apoptosis through activation of cJun N-terminal kinase, suppression of Akt pathway, and downregulation of antiapoptotic gene products. Biochem Pharmacol. 2007;74(1):118-30.

18. Goyal C, Ahuja M, Sharma SK. Preparation and evaluation of anti-inflammatory activity of gugulipid-loaded proniosomal gel. Acta Pol Pharm - Drug Res. 2011;68(1):147-50.

19. Ichikawa H, Aggarwal BB. Guggulsterone inhibits osteoclastogenesis induced by receptor activator of nuclear factor-kappa B ligand and by tumor cells by suppressing nuclear factor-kappa B activation. Clin Cancer Res. 2006;12(2):662-8.

20. Shodia S, Aggarwal BB. Guggulsterone inhibits NF-??B and I??B?? kinase activation, suppresses expression of antiapoptotic gene products, and enhances apoptosis. J Biol Chem. 2004;279(45):47148-58.

21. Suzuki E, Mellins ED, Gershwin ME, Nestle FO, Adamopoulos IE. The IL-23/IL17 axis in psoriatic arthritis. Autoimmun Rev. 2014;13(4-5):496-502.

22. Zealand N. Metabolic Isolated Properties From Adult Mouse Preparation of Suspensions. 1962;(3):1-8.

23. van der Fits L, Mourits S, Voerman JSA, Kant M, Boon L, Laman JD, et al. Imiquimod-Induced Psoriasis-Like Skin Inflammation in Mice Is Mediated via the IL-23/IL-17 Axis. J Immunol. 2009 ; 182 (9)
: $5836-45$.

24. Zhang S, Liu X, Mei L, Wang H, Fang F. Epigallocatechin - 3 - gallate (EGCG) inhibits imiquimod-induced psoriasis - like inflammation of $\mathrm{BALB} / \mathrm{c}$ mice. BMC Complement Altern Med . 2016;16(1):1-11.

25. Van der Fits L, Mourits ST cumulative score (erythema plus scaling plus thickening) served as a measure of the severity of inflammation (scale 0-12), Voerman JSA, Kant M, Boon L, Laman JD, et al. Imiquimod-Induced Psoriasis-Like Skin Inflammation in Mice Is Mediated via the IL-23/IL-17 Axis. J Immunol. 2009;182(9):5836-45.

26. Nakajima K, Kanda T, Takaishi M, Shiga T, Miyoshi K, Nakajima $\mathrm{H}$, et al. Distinct Roles of IL-23 and IL-17 in the Development of Psoriasis-Like Lesions in a Mouse Model. J Immunol. 2011;186(7):4481-9.

27. Cooler L, Cooler L. Serum Collection Protocol. :4-6.

28. Luna LG. Preparation of Tissue. Man Histol. 1968;(April):1-36.

29. Ute L., Paola D.M., Gayathri K. P, et al Identification of a novel pro-inflammatory human skin-homing $\mathrm{V} \gamma 9 \mathrm{~V} \delta 2 \mathrm{~T}$ cell subset with a potential role in psoriasis. J Immunol. 2011; 187(5): 2783-2793.

30. Cowan G. Statistical Data Analysis. Metall Ital. 2017;109(1):5-10.

31. Imiquimod RA. Review ArticleImiquimod applied topically] a novel immune response modi_er and new class of drug. $1999 ; 1$ 14. Available from: papers $2: / /$ publication /uuid /B5C54702-C3BA-411B-86E04B9C541F4F7F

32. Walter A, Schäfer M, Cecconi V, Matter C, Urosevic-Maiwald $\mathrm{M}$, Belloni $\mathrm{B}$, et al. Aldara activates TLR7-independent immune defence. Nat Commun. 2013;4:113.

33. Libert C, Vandenbroucke RE. An inflammatory triangle in psoriasis: TNF, type I IFNs and IL-17. Cytokine Growth Factor Rev. 2015;26(1):25-33.

34. Levine D, Gottlieb A. Evaluation and Management of Psoriasis: An Internist's Guide. Med Clin North Am. 2009 ;93 (6) :1291 -303. 